\title{
FIXED POINT THEORY OF MÖNCH TYPE FOR WEAKLY SEQUENTIALLY UPPER SEMICONTINUOUS MAPS
}

\author{
Donal O'REgan
}

A variety of fixed point results are presented for weakly sequentially upper semicontinuous maps. In addition an existence result is established for differential equations in Banach spaces relative to the weak topology.

\section{INTRODUCTION}

New fixed point theorems are presented for weakly sequentially continuous (and more generally weakly sequentially upper semicontinuous) maps between Banach spaces (or more generally metrisable locally convex spaces). In particular we extend Emmanuele's and other fixed point theory in the literature $[5,13,14]$. Also we present an analogue of Mönch's fixed point theorem [11] in the weak topology setting for weakly sequentially continuous maps. The paper will be divided into three main sections. Section 2 (respectively Section 4) discusses fixed point theory for single valued (respectively multivalued) maps whereas in Section 3 we use the theory developed in Section 2 to establish the existence of weak solutions to differential equations.

\section{Single VALUED MAPS}

We shall establish two new fixed point results in this section. However before we do so we present a well known result from the literature which will be used throughout this section (for completness we include its proof).

THEOREM 2.1. Let $E$ be a metrisable locally convex linear topological space with $Q$ a weakly compact subset of $E$. Suppose $F: Q \rightarrow E$ is weakly sequentially continuous. Then $F: Q \rightarrow E$ is weakly continuous.

Proof: Let $A$ be a weakly closed subset of $E$. We first show $F^{-1}(A)$ is weakly sequentially closed in $Q$. To see this let $y_{n} \in F^{-1}(A)$ and $y_{n} \rightarrow y$ (here $\rightarrow$ denotes weak convergence). Then $F\left(y_{n}\right) \rightarrow F(y)$. Also $F\left(y_{n}\right) \in A$ and $A$ weakly closed implies $F(y) \in A$, that is, $y \in F^{-1}(A)$. Thus $F^{-1}(A)$ is weakly sequentially closed. Now since $Q$ is weakly compact we have $\overline{F^{-1}(A)^{w}}$ weakly compact. Let $x \in \overline{F^{-1}(A)^{w}}$. The

Received 20th August, 1999

Copyright Clearance Centre, Inc. Serial-fee code: 0004-9727/00 \$A2.00+0.00. 
Eberlein-Smulian Theorem [4, p.549] guarantees that there is a sequence $x_{n} \in F^{-1}(A)$ with $x_{n} \rightarrow x$. Since $F^{-1}(A)$ is weakly sequentially closed we have $x \in F^{-1}(A)$, that is, $\overline{F^{-1}(A)^{w}}=F^{-1}(A)$. Thus $F^{-1}(A)$ is weakly closed.

Our first result is the analogue of Mönch's fixed point theorem in the weak topology setting.

THEOREM 2.2. Let $E$ be a Banach space (or more generally a quasicomplete metrisable locally convex linear topological space), $Q$ a closed, convex subset of $E$ and $x_{0} \in Q$. Suppose there is a weakly sequentially continuous map $F: Q \rightarrow Q$ and assume the following properties hold:

$$
\left\{\begin{array}{l}
C \subseteq Q \text { is countable and } \overline{C^{w}} \subseteq \overline{c o}\left(\left\{x_{0}\right\} \cup F\left(\overline{C^{w}}\right)\right) \\
\text { implies } \overline{C^{w}} \text { is weakly compact }
\end{array}\right.
$$

and

$$
\left\{\begin{array}{l}
\text { for any relatively weakly compact subset } A \text { of } E \text { there } \\
\text { exists a countable set } B \text { of } E \text { with } \overline{B^{w}}=\overline{A^{w}}
\end{array}\right.
$$

Then $F$ has a fixed point in $Q$.

REMARK 2.2. If $E$ is a Banach space and $E^{\star}$ (the dual of $E$ ) is separable then (2.2) is true. To see this recall if $K$ is a weakly compact subset of $E$ then $K$ with the relative weak topology is metrisable. This together with the fact that compact metric spaces are separable yields (2.2).

Proof: Let

$$
D_{0}=\left\{x_{0}\right\} \text { and } D_{n}=c o\left(\left\{x_{0}\right\} \cup F\left(D_{n-1}\right)\right) \text { for } n=1,2, \ldots .
$$

We claim $D_{n}$ is relatively weakly compact for $n=0,1, \ldots$ Certainly it is true if $n=0$. Now suppose $D_{k}$ is relatively weakly compact for some $k \in\{1,2, \ldots\}$. Notice from Theorem 2.1 that

$$
F: \overline{D_{k}^{w}} \rightarrow E \text { is weakly continuous, }
$$

and as a result

$$
F\left(\overline{D_{k}^{w}}\right) \text { is weakly compact. }
$$

The Krein-Smulian Theorem [3, p.434]; [6, p.82] guarantees that $D_{k+1}$ is relatively weakly compact.

From (2.2) there exists a sequence of countable sets $\left\{C_{n}\right\}_{0}^{\infty}$ with $\overline{C_{n}^{\bar{w}}}=\overline{D_{n}^{w}}$ for $n=0,1, \ldots$ Let

$$
D=\bigcup_{n=0}^{\infty} D_{n} \text { and } C=\bigcup_{n=0}^{\infty} C_{n} \text {. }
$$


Note that $D$ is convex, since $D_{n-1} \subseteq D_{n}$ for $n=1,2, \ldots$ It is immediate since $\left(D_{n}\right)$ is increasing that

$$
D=\bigcup_{n=0}^{\infty} D_{n}=\bigcup_{n=1}^{\infty} c o\left(\left\{x_{0}\right\} \cup F\left(D_{n-1}\right)\right)=c o\left(\left\{x_{0}\right\} \cup F(D)\right)
$$

and so $[15$, p.66] guarantees that

$$
\overline{D^{w}}(=\bar{D})=\overline{c o}\left(\left\{x_{0}\right\} \cup F(D)\right) .
$$

Also since

$$
\bigcup_{n=0}^{\infty} D_{n} \subseteq \bigcup_{n=0}^{\infty} \overline{D_{n}^{w}} \subseteq \overline{\bigcup_{n=0}^{\infty} D_{n}{ }^{w}}
$$

we have

$$
\overline{\bigcup_{n=0}^{\infty} \overline{D_{n}^{w}}}=\overline{\bigcup_{n=0}^{\infty} D_{n}{ }^{w}}=\overline{D^{w}} \text { and } \overline{\bigcup_{n=0}^{\infty} \overline{D_{n}^{w}}}=\overline{\bigcup_{n=0}^{\infty} \overline{C_{n}^{w}}}=\overline{\bigcup_{n=0}^{\infty} C_{n}{ }^{w}}=\overline{C^{w}} \text {. }
$$

Now (2.3) and (2.4) imply

$$
\overline{C^{w}}=\overline{D^{w}}=\overline{c o}\left(F(D) \cup\left\{x_{0}\right\}\right) \subseteq \overline{c o}\left(F\left(\overline{D^{w}}\right) \cup\left\{x_{0}\right\}\right)=\overline{c o}\left(F\left(\overline{C^{w}}\right) \cup\left\{x_{0}\right\}\right) .
$$

This together with (2.1) guarantees that $\overline{C^{w}}$ is weakly compact. Hence $\overline{D^{w}}$ is weakly compact. From Theorem 2.1 we have that

$$
F: \overline{D^{w}} \rightarrow E \text { is weakly continuous. }
$$

Also (2.3) implies $F(D) \subseteq \overline{D^{w}}$ and so

$$
\overline{F(D)^{w}} \subseteq \overline{D^{w}} .
$$

Now (2.5) and (2.6) (note the weak closure of $F(D)$ (respectively $D$ ) in $\overline{D^{w}}$ equals the weak closure of $F(D)$ (respectively $D$ ) in $E$ ) gives

$$
F\left(\overline{D^{w}}\right) \subseteq \overline{F(D)^{w}} \subseteq \overline{D^{w}} .
$$

We may apply the Schauder-Tychonoff Theorem (consider $E$ with the weak topology and note $F: \overline{D^{w}} \rightarrow \overline{D^{w}}$ is continuous with $\overline{D^{w}}$ compact) to deduce that $F$ has a fixed point in $\overline{D^{w}}$.

We now present a fixed point result when (2.2) is not assumed. As one would expect (2.1) needs to be adjusted also.

TheOREM 2.3. Let $E$ be a Banach space (or more generally a metrisable locally convex linear topological space), $Q$ a closed, convex subset of $E$ and $x_{0} \in Q$. Suppose 
there is a weakly sequentially continuous map $F: Q \rightarrow Q$ with the following property holding:

$$
\left\{\begin{array}{l}
A \subseteq Q \text { and } \bar{A}=\overline{c o}\left(\left\{x_{0}\right\} \cup F(A)\right) \\
\text { implies } \overline{A^{w}} \text { is weakly compact. }
\end{array}\right.
$$

Then $F$ has a fixed point in $Q$.

Proof: Let

$$
D_{0}=\left\{x_{0}\right\}, D_{n}=c o\left(\left\{x_{0}\right\} \cup F\left(D_{n-1}\right)\right) \text { for } n=1,2, \ldots, \text { and } D=\bigcup_{n=0}^{\infty} D_{n} .
$$

As in Theorem 2.2 we have

$$
\bar{D}=\overline{D^{w}}=\overline{c o}\left(F(D) \cup\left\{x_{0}\right\}\right) .
$$

Now (2.7) implies $\overline{D^{w}}$ is weakly compact. Note also that $F(D) \subseteq \overline{D^{w}}$ and so $\overline{F(D)^{w}} \subseteq$ $\overline{D^{w}}$. In addition Theorem 2.1 guarantees that $F: \overline{D^{w}} \rightarrow E$ is weakly continuous, so

$$
F\left(\overline{D^{w}}\right) \subseteq \overline{F(D)^{w}} \subseteq \overline{D^{w}} .
$$

Apply the Schauder-Tychonoff Theorem to deduce the result.

\section{Application}

We begin with a discussion of the operator equation

$$
x(t)=F x(t) \text { on }[0, T] .
$$

Solutions to (3.1) will be sought in $C([0, T], E)$.

TheOREM 3.1. Let $E$ be a Banach space with $Q$ a nonempty, bounded, closed, convex, equicontinuous subset of $C([0, T], E)$. Suppose $F: Q \rightarrow Q$ is wk-sequentially continuous (that is, if for any sequence $\left(x_{n}\right)$ in $Q$ with $x_{n}(t) \rightarrow x(t)$ in $(E, w)$ for each $t \in[0, T]$, then $F x_{n}(t) \rightarrow F x(t)$ in $(E, w)$ for each $\left.t \in[0, T]\right)$ and assume (2.7) holds. Then (3.1) has a solution in $Q$.

Proof: The argument in [12, p.103] guarantees that $F: Q \rightarrow Q$ is weakly sequentially continuous so the result follows from Theorem 2.3 .

We next gather together some facts that will be needed in this section. Let $\Omega_{E}$ be the bounded subsets of a Banach space $E$ and let $K^{w}$ be the family of all weakly compact subsets of $E$. Also let $B_{E}$ be the closed unit ball of $E$. The De Blasi measure of weak noncompactness is the map $\beta: \Omega_{E} \rightarrow[0, \infty)$ defined by

$$
\beta(X)=\inf \left\{t>0: \exists Y \in K^{w} \text { with } X \subseteq Y+t B_{E}\right\}: \text { here } X \in \Omega_{E} .
$$


We now state the following well known result [10].

THEOREM 3.2. Let $H \subseteq C([0, T], E)$ be bounded and equicontinuous. Then

$$
\beta(H)=\sup _{t \in[0, T]} \beta(H(t))=\beta(H[0, T])
$$

and the function $t \mapsto \beta(H(t))$ is continuous; here

$$
H(t)=\{\phi(t): \phi \in H\} \text { and } H[0, T]=\bigcup_{t \in\{0, T]}\{\phi(t): \phi \in H\} .
$$

We now discuss a special case of (3.1), namely

$$
y(t)=x_{0}+\int_{0}^{t} f(s, y(s)) d s \text { for } t \in[0, T]
$$

here $x_{0} \in E$ and $E=(E,||$.$) is a real Banach space. Assume that the following conditions$ hold:

$$
\left\{\begin{array}{l}
\text { for each } t \in[0, T], f_{t}=f(t, .) \text { is weakly sequentially continuous } \\
\text { (that is, for each } t \in[0, T], \text { and for each convergent sequence }\left(x_{n}\right), \\
\text { the sequence } f_{t}\left(x_{n}\right) \text { is weakly convergent) }
\end{array}\right.
$$

$$
\text { for each continuous } y:[0, T] \rightarrow E, f(., y(.)) \text { is Pettis integrable on }[0, T] \text {, }
$$

and

$$
\left\{\begin{array}{l}
\text { for any } r>0 \text { there exists } h_{r} \in L^{1}[0, T] \text { with }|f(t, y)| \leqslant h_{r}(t) \\
\text { for almost all } t \in[0, T] \text { and all } y \in E \text { with }|y| \leqslant r
\end{array}\right.
$$

Define the operator $F$ by

$$
F x(t)=x_{0}+\int_{0}^{t} f(s, x(s)) d s
$$

A standard argument $[12$, p.103] guarantees that

$$
F: C([0, T], E) \rightarrow C([0, T], E)
$$

We say $w:[0, \infty) \rightarrow[0, \infty)$ is a Kamke function if the unique solution to the integral inequality

$$
u(t) \leqslant \int_{0}^{t} w(u(\tau)) d \tau, \quad t \in[0, T]
$$

which satisfies $u(0)=0$ is $u \equiv 0$. 
TheOrem 3.3. Let $E$ be a Banach space with $Q$ a nonempty, bounded, closed, convex, equicontinuous subset of $C([0, T], E)$. Suppose (3.3), (3.4) and (3.5) hold. Also assume

$$
\beta(f([0, T] \times X)) \leqslant w(\beta(X)) \text { for all bounded subsets } X \text { of } E
$$

is satisfied; here $w$ is a Kamke function. Let $F$ be as defined in (3.6) and assume $F: Q \rightarrow Q$. Then (3.2) has a solution in $Q$.

ProOF: We shall apply Theorem 3.1. First we show $F: Q \rightarrow Q$ is $w k$-sequentially continuous. Let $\left(x_{n}\right)$ be a sequence in $Q$ and let $x_{n}(t) \rightarrow x(t)$ in $(E, w)$ for each $t \in[0, T]$. Fix $t \in(0, T]$. Since $f_{t}$ is weakly sequentially continuous, the Lebesgue dominated convergence theorem for the Pettis integral [7, Corollary 4$]$ implies for each $\phi \in E^{\star}$ that

$$
\phi\left(F x_{n}(t)\right) \rightarrow \phi(F x(t)) .
$$

We can do this for each $t \in[0, T]$ and so $F: Q \rightarrow Q$ is $w k$-sequentially continuous. It remains to show (2.7). Let $x^{\star} \in Q$ and $\bar{C}=\overline{c o}\left(\left\{x^{\star}\right\} \cup F(C)\right)$ for some $C \subseteq Q$. We must show $C$ is relatively weakly compact. Notice from Theorem 3.2 that the function

$$
v: t \rightarrow \beta(C(t)) \text { is continuous on }[0, T] \text {. }
$$

For fixed $t \in(0, T]$ divide $[0, t]$ into $m$ parts: $0=t_{0}<t_{1}<\ldots<t_{m}=t$, where $t_{i}=i t / m$ for $i=0,1, \ldots, m$. Let

$$
C\left[t_{i-1}, t_{i}\right]=\left\{u(s): u \in C \text { and } t_{i-1} \leqslant s \leqslant t_{i}\right\} .
$$

There exists (by Theorem 3.2 and the continuity of $v$ ) $s_{i} \in\left[t_{i-1}, t_{i}\right]$ with

$$
\beta\left(C\left[t_{i-1}, t_{i}\right]\right)=\sup \left\{\beta(C(s)): t_{i-1} \leqslant s \leqslant t_{i}\right\}=v\left(s_{i}\right) .
$$

Also by the Pettis integral mean value theorem we obtain for $u \in C$,

$$
F u(t)=x_{0}+\sum_{i=0}^{m-1} \int_{t_{i}}^{t_{i+1}} f(s, u(s)) d s \in x_{0}+\sum_{i=0}^{m-1}\left(t_{i+1}-t_{i}\right) \overline{c o}\left(f\left([0, T] \times C\left[t_{i}, t_{i+1}\right]\right)\right),
$$

and so

$$
F C(t) \subseteq x_{0}+\sum_{i=0}^{m-1}\left(t_{i+1}-t_{i}\right) \overline{c o}\left(f\left([0, T] \times C\left[t_{i}, t_{i+1}\right]\right)\right)
$$

Now

$$
\begin{aligned}
\beta(F C(t)) & \leqslant \sum_{i=0}^{m-1}\left(t_{i+1}-t_{i}\right) \beta\left(\overline{c o}\left(f\left([0, T] \times C\left[t_{i}, t_{i+1}\right]\right)\right)\right) \\
& =\sum_{i=0}^{m-1}\left(t_{i+1}-t_{i}\right) \beta\left(f\left([0, T] \times C\left[t_{i}, t_{i+1}\right]\right)\right) \\
& \leqslant \sum_{i=0}^{m-1}\left(t_{i+1}-t_{i}\right) w\left(\beta\left(C\left[t_{i}, t_{i+1}\right]\right)\right) \\
& =\sum_{i=0}^{m-1}\left(t_{i+1}-t_{i}\right) w\left(v\left(s_{i}\right)\right)
\end{aligned}
$$


using (3.7) and (3.8). Let $m \rightarrow \infty$ and we have

$$
\sum_{i=0}^{m-1}\left(t_{i+1}-t_{i}\right) w\left(v\left(s_{i}\right)\right) \rightarrow \int_{0}^{t} w(v(s)) d s
$$

so

$$
\beta(F C(t)) \leqslant \int_{0}^{t} w(v(s)) d s \text { for } t \in[0, T] .
$$

Since $\bar{C}=\overline{c o}\left(\left\{x^{\star}\right\} \cup F(C)\right)$ we have for $t \in[0, T]$ that

$$
\beta(C(t)) \leqslant \beta(F C(t))
$$

and this together with (3.9) yields

$$
v(t)=\beta(C(t)) \leqslant \int_{0}^{t} w(v(s)) d s .
$$

Now since $w$ is a Kamke function we get $v(t)=0$ for each $t \in[0, T]$, that is, $\beta(C(t))=0$ for each $t \in[0, T]$. This together with Theorem 3.2 implies $C$ is relatively weakly compact. Thus (2.7) holds. Our result follows from Theorem 3.1.

We now use Theorem 3.3 to establish a general existence result for (3.6).

THEOREM 3.4. Let E be a Banach space and suppose (3.3), (3.4) and (3.7) hold. In addition assume

$$
\left\{\begin{array}{l}
\text { there exists } \alpha \in L^{1}[0, T] \text { and } \psi:[0, \infty) \rightarrow(0, \infty) \text { a nondecreasing continuous } \\
\text { function with }|f(s, u)| \leqslant \alpha(s) \psi(|u|) \text { for almost all } s \in[0, T] \text { and all } u \in E
\end{array}\right.
$$

and

$$
\int_{0}^{T} \alpha(s) d s<\int_{\left|x_{0}\right|}^{\infty} \frac{d x}{\psi(x)}
$$

are satisfied. Then (3.2) has a solution in $C([0, T], E)$.

Proof: Let

$$
\begin{aligned}
Q=\{y \in C([0, T], E): & |y(t)| \leqslant b(t) \text { for } t \in[0, T] \text { and } \\
& |y(t)-y(s)| \leqslant b(t)-b(s) \text { for } t, s \in[0, T]\}
\end{aligned}
$$

where

$$
b(t)=I^{-1}\left(\int_{0}^{t} \alpha(s) d s\right) \text { and } I(z)=\int_{\left|x_{0}\right|}^{z} \frac{d x}{\psi(x)} .
$$

Notice $Q$ is a closed, convex, bounded, equicontinuous subset of $C([0, T], E)$. Let $F$ be as defined in (3.6). A standard argument $[12, \mathrm{p} .104]$ guarantees that $F: Q \rightarrow Q$. The result follows from Theorem 3.3. 


\section{Multivalued Maps}

We begin with the analogue of Theorem 2.1 in the multivalued setting.

THEOREM 4.1. Let $E$ be a metrisable locally convex linear topological space with $Q$ a weakly compact subset of $E$. Suppose $F: Q \rightarrow C(E)$ (here $C(E)$ denotes the family of nonempty, closed, convex subsets of $E$ ) is a weakly sequentially upper semicontinuous map. Then $F: Q \rightarrow C(E)$ is weakly upper semicontinuous.

REMARK 4.1. Note $F: Q \rightarrow C(E)$ is weakly sequentially upper semicontinuous if for any weakly closed set $A$ of $E, F^{-1}(A)$ is weakly sequentially closed.

Proof: Let $A$ be a weakly closed subset of $E$. Then since $F: Q \rightarrow C(E)$ is weakly sequentially upper semicontinuous we have that $F^{-1}(A)$ is weakly sequentially closed. Now since $Q$ is weakly compact we have $\overline{F^{-1}(A)^{w}}$ weakly compact. Let $x \in \overline{F^{-1}(A)^{w}}$. The Eberlein-Smulian Theorem [4, p.549] guarantees that there is a sequence $x_{n} \in F^{-1}(A)$ with $x_{n} \rightarrow x$. Now $x \in F^{-1}(A)$ since $F^{-1}(A)$ is weakly sequentially closed. Thus $\overline{F^{-1}(A)^{w}}=F^{-1}(A)$ so $F^{-1}(A)$ is weakly closed.

The following well known result of Himmelberg [8, p.206] will be used in this section (alternatively we could use Ky Fan's fixed point theorem).

THEOREM 4.2. Let $U$ be a nonempty, convex subset of a Hausdorff locally convex linear topological space $E$. Let $F: U \rightarrow U$ be an upper semicontinuous multifunction such that $F(x)$ is closed and convex for all $x \in U$ and $F(U)$ is contained in a compact subset $Q$ of $U$. Then $F$ has a fixed point.

We begin by presenting the analogue of Theorem 2.3 for multivalued maps.

THEOREM 4.3. Let $E$ be a Banach space (or more generally a metrisable locally convex linear topological space), $Q$ a closed, convex subset of $E$ and $x_{0} \in Q$. Suppose $F: Q \rightarrow C(Q)$ is a weakly sequentially closed map (that is, has weakly sequentially closed graph) with (2.7) holding. In addition assume

$$
F\left(\overline{A^{w}}\right) \subseteq \overline{F(A)^{w}} \text { for every relatively weakly compact subset } A \text { of } Q .
$$

Then $F$ has a fixed point in $Q$.

ProOF: Let

$$
D_{0}=\left\{x_{0}\right\}, D_{n}=c o\left(\left\{x_{0}\right\} \cup F\left(D_{n-1}\right)\right) \text { for } n=1,2, \ldots, \text { and } D=\bigcup_{n=0}^{\infty} D_{n} \text {. }
$$

As in Theorem 2.2 we have

$$
D=\operatorname{co}\left(F(D) \cup\left\{x_{0}\right\}\right)
$$

and

$$
\bar{D}=\overline{D^{w}}=\overline{c o}\left(F(D) \cup\left\{x_{0}\right\}\right)
$$


Now (2.7) and (4.3) imply that $\overline{D^{w}}$ is weakly compact. Also (4.3) implies $F(D) \subseteq \overline{D^{w}}$, and so $\overline{F(D)^{w}} \subseteq \overline{D^{w}}$. This together with (4.1) yields $F\left(\overline{D^{w}}\right) \subseteq \overline{D^{w}}$. Thus

$$
F: \overline{D^{w}} \rightarrow C\left(\overline{D^{w}}\right) \text { with } F \text { a weakly sequentially closed map. }
$$

The result follows from [14, Theorem 2.2] (the argument in [14] shows $F$ is weakly upper semicontinuous so $F$ has a fixed point by Theorem 4.2).

We now discuss the analogue of Theorem 2.2. Two results will be presented.

THEOREM 4.4. Let $E$ be a Banach space (or more generally a quasicomplete metrisable locally convex linear topological space), $Q$ a closed, convex subset of $E$ and $x_{0} \in Q$. Suppose there is a weakly sequentially upper semicontinuous map $F: Q \rightarrow C(Q)$ with (2.1) and (2.2) holding. In addition assume (4.1) is satisfied. Then $F$ has a fixed point in $Q$.

Proof: Let $D_{n}$ be as in Theorem 2.2. Suppose $D_{k}$ is relatively weakly compact for some $k \in\{1,2, \ldots\}$. Notice from Theorem 4.1 that

$$
F: \overline{D_{k}^{w}} \rightarrow C(E) \text { is weakly upper semicontinuous, }
$$

and so $[1$, p.464] guarantees that

$$
F\left(\overline{D_{k}^{w}}\right) \text { is weakly compact. }
$$

The Krein-Smulian Theorem [6, p.82] guarantees that $D_{k+1}$ is relatively weakly compact.

Let $C_{n}, D, C$ be as in Theorem 2.2 and note (as in Theorem 2.2) that

$$
D=\operatorname{co}\left(F(D) \cup\left\{x_{0}\right\}\right)
$$

and

$$
\bar{D}=\overline{D^{w}}=\overline{c o}\left(F(D) \cup\left\{x_{0}\right\}\right) \text { and } \overline{D^{w}}=\overline{C^{w}}
$$

Consequently

$$
\overline{C^{w}}=\overline{D^{w}}=\overline{c o}\left(F(D) \cup\left\{x_{0}\right\}\right) \subseteq \overline{c o}\left(F\left(\overline{D^{w}}\right) \cup\left\{x_{0}\right\}\right)=\overline{c o}\left(F\left(\overline{C^{w}}\right) \cup\left\{x_{0}\right\}\right) .
$$

Now (2.1) guarantees that $\overline{C^{w}}$ (and so $\overline{D^{w}}$ ) is weakly compact. Also notice from (4.4) that

$$
F: D \rightarrow C(D) \text { with } F(D) \subseteq \overline{D^{w}} \text { and } \overline{D^{w}} \text { is weakly compact. }
$$

Theorem 4.1 now implies that $F: D \rightarrow C(D)$ is weakly upper semicontinuous. In addition (4.1) guarantees that $F\left(\overline{D^{w}}\right) \subseteq \overline{F(D)^{w}} \subseteq \overline{D^{w}}$. Now apply Theorem 4.2.

In our next result we replace (2.1) with a less restrictive condition.

THEOREM 4.5. Let $E$ be a Banach space (or more generally a quasicomplete metrisable locally convex linear topological space), $Q$ a closed, convex subset of $E$ and 
$x_{0} \in Q$. Suppose $F: Q \rightarrow C(Q)$ is a weakly sequentially upper semicontinuous map with (2.2) holding. In addition suppose

$$
\left\{\begin{array}{l}
C \subseteq Q \text { is countable and } \overline{C^{w}}=\overline{c o}\left(\left\{x_{0}\right\} \cup F(C)\right) \\
\text { implies } C \text { is relatively weakly compact }
\end{array}\right.
$$

and

$$
F\left(\overline{A^{w}}\right) \subseteq \overline{F(A)^{w}} \text { for any subset } A \text { of } Q
$$

are satisfied. Then $F$ has a fixed point.

REMARK 4.2. Notice that $\overline{c o}\left(\left\{x_{0}\right\} \cup F(C)\right)$ could be replaced by $\overline{c o}\left(\left\{x_{0}\right\} \cup F\left(\overline{C^{w}}\right)\right)$ in (4.6).

Proof: Let $D_{n}$ be as in Theorem 2.2. As in Theorem 4.4 we have that $D_{n}$ is relatively weakly compact for each $n=0,1, \ldots$ Let $C_{n}, D, C$ be as in Theorem 2.2 and note

$$
\bar{D}=\overline{D^{w}}=\overline{c o}\left(F(D) \cup\left\{x_{0}\right\}\right) \text { and } \overline{D^{w}}=\overline{C^{w}} .
$$

In addition (4.7) implies

$$
F(D) \cup\left\{x_{0}\right\} \subseteq F\left(\overline{D^{w}}\right) \cup\left\{x_{0}\right\} \subseteq \overline{F(D) \cup\left\{x_{0}\right\}^{w}} \subseteq \overline{c o}\left(F(D) \cup\left\{x_{0}\right\}\right)
$$

and so

$$
\overline{c o}\left(F(D) \cup\left\{x_{0}\right\}\right)=\overline{c o}\left(F\left(\overline{D^{w}}\right) \cup\left\{x_{0}\right\}\right) .
$$

Then (4.8) and (4.9) imply

$\overline{C^{w}}=\overline{D^{w}}=\overline{c o}\left(F(D) \cup\left\{x_{0}\right\}\right)=\overline{c o}\left(F\left(\overline{D^{w}}\right) \cup\left\{x_{0}\right\}\right)=\overline{c o}\left(F\left(\overline{C^{w}}\right) \cup\left\{x_{0}\right\}\right)=\overline{c o}\left(F(C) \cup\left\{x_{0}\right\}\right)$.

Now (4.6) guarantees that $\overline{C^{w}}$ (and so $\overline{D^{w}}$ ) is weakly compact. Also $F\left(\overline{D^{w}}\right) \subseteq \overline{D^{w}}$ and Theorem 4.1 guarantees that $F: \overline{D^{w}} \rightarrow C\left(\overline{D^{w}}\right)$ is weakly upper semicontinuous. Now the result of the theorem follows from Theorem 4.2.

\section{REFERENCES}

[1] C.D. Aliprantis and K.C. Border, Infinite dimensional analysis (Springer-Verlag, Berlin, Heidelberg, New York, 1985).

[2] M. Cichon, 'On bounded weak solutions of a nonlinear differential equation in a Banach space', Funct. Approx. Comment. Math. 21 (1992), 27-35.

[3] N. Dunford and J.T. Schwartz, Linear operators; Part I: General theory, A Wiley Interscience Publication (John Wiley and Sons Inc., New York, London, 1958). 
[4] R.E. Edwards, Functional analysis, theory and applications (Holt, Rinehart and Winston, New York, 1965).

[5] G. Emmanuele, 'Measures of weak noncompactness and fixed point theorems', Bull. Math. Soc. Sci. Math. R.S. Roumanie 25 (1981), 353-358.

[6] K. Floret, Weakly compact sets (Springer-Verlag, Berlin, Heidelberg, New York, 1980).

[7] R.F. Geitz, 'Pettis integration', Proc. Amer. Math. Soc. 82 (1981), 81-86.

[8] C.J. Himmelberg, 'Fixed points of compact multifunctions', J. Math. Anal. Appl. 38 (1972), 205-207.

[9] I. Kubiaczyk and S. Szufla, 'Knaeser's theorem for weak solutions of ordinary differential equations in Banach spaces', Publ. Inst. Math. (Beograd) 32 (1982), 99-103.

[10] A.R. Mitchell and C.K.L. Smith, 'An existence theorem for weak solutions of differential equations in Banach spaces', in Nonlinear equations in abstract spaces, (V. Lakshmikantham, Editor) (Academic Press, New York, 1978), pp. 387-404.

[11] H. Mönch, Boundary value problems for nonlinear ordinary differential equations of second order in Banach spaces, Nonlinear Anal. 4 (1980), 985-999.

[12] D. O'Regan, 'Weak solutions of ordinary differential equations in Banach spaces', Appl. Math. Lett. 12 (1999), 101-105.

[13] D. O'Regan, 'Fixed point theory for weakly contractive maps with applications to operator equations in Banach spaces relative to the weak topology', Z. Anal. Anwendungen 17 (1998), 281-296.

[14] D. O'Regan, 'Fixed point theorems for weakly sequentially closed maps', Arch. Math. (Brno) (to appear).

[15] W. Rudin, Functional analysis (McGraw Hill, New York, 1973).

Department Of Mathematics

National University of Ireland

Galway

Ireland 